\title{
The Development of Diagnostics Tools and Techniques in the Isolation and Detection of Fungal Pathogens
}

Ousman Bajinka*, Yığıt Terzi and Fusun Ucar

Department of Microbiology, Ege University, Izmir, Turkey

*Corresponding author: Ousman Bajinka, Department of Microbiology, Ege University, Izmir, Turkey, Tel: +905061792543; E-mail: bajinkaousman@gmail.com

Received date: December 04, 2017; Accepted date: December 14, 2017; Published date: December 21, 2017

Copyright: ( 2017 Bajinka O, et al. This is an open-access article distributed under the terms of the Creative Commons Attribution License; which permits unrestricted use; distribution; and reproduction in any medium; provided the original author and source are credited.

\begin{abstract}
Until of the recent, the use of molecular tools in diagnostic mycology laboratories supersedes the old conventional methods. The diagnostics laboratories consider two important parameters; sensitivity and specificity, and the use of PCR and it is related have it all. This review went through the timeline of how fungal pathogen detection techniques have developed and still in the progress. The most recent other review articles and research papers were selected at random and critical analyses are made on the previous studies and papers. It is ascertain that molecular tools are more sensitive and specific and hence accurate results are achieved. It is clear that even though molecular techniques are reliable enough, the methods and the purity of DNA extraction and the amount respectively determines the fingerprint that gives identity to the respective pathogens.
\end{abstract}

Keywords: Invasive fungal infections; Specificity and sensitivity Pathogens; Polymerase chain reaction

\section{Introduction}

Fungi unlike bacteria and virus are considered to be less pathogenic to human and plants. Among the approximated 300,000 species, only a few are pathogenic. They live and survive on most plants and animals as commensals or normal body flora. However, if the immune system of these individuals is compromised, some strains can be pathogenic [1-3].

Fungi belong to the kingdom that since pre-history has been playing a very important role in the ecology through the breaking down of dead organic matters to keep the life cycle. In addition, fungi are of great economic importance. They cause considerable diseases to both plants and animals that are but not limited to; ringworms, athlete's foot in animals and smuts, rusts, rots of leaf, roots and stem in plants. They sometimes while depending on each other, an example can the symbiotic relationship shown by mycorrhizae (symbiotic association between a fungus and the roots of a vascular host plant). Despite the economic lost caused by fungi through infecting the plants and animals, until today fungi have been providing the medicinal industries with drugs without number. One of the common ones includes penicillin as antibiotics $[1,2]$.

Furthermore, those very palatable cuisines are of fungi ingredients that can be found in mushrooms, truffles, champagne, beer and morels etc. Due to the genetic similarities between fungi and animals, their disease causing mechanisms are difficult to study except for the yeast fungi type that serves as human model organisms [4-6].

The most common invasive infections are caused by Candida spp. and Aspergillus. Fungal infections are normally believed to be opportunistic in nature and are very rare infections to both human and plants. However, with the intense study on its diseases mechanisms, the epidemiology and the revolutionary history of fungal taxonomy, a light has been shed on its virulence on other organism [2-6].
The reasons for the outspread of fungal diseases are numerous and are now being comprehended by the pathologists. Majority of the occurrence are due to the immune compromised statues of patients and this made them very prone to fungal infections. These are people whose immune systems are weakening due to conditions like impaired host defense mechanisms. Impaired host defense are normally due to some deadly viral infections that weaken the cells to back against foreign bodies. One of these is Human immunodeficiency virus (HIV virus). Also due to organ transplants and leukaemia (blood disorder that comprises group of cancers that usually begin in the bone marrow and result in high numbers of abnormal white blood cells), clinical procedures such as surgery, injections, radiation, the use of catheters, chemotherapy to name but a few. Unfortunately most of these procedures are unavoidable and hence the dramatically growing of fungal infections [2-6].

Collection and transportation of these samples for the identification of potential pathogenic agents also contribute immensely to achieving a better result. A delay in transporting samples affect their quality and hence forth a controversy results. For some, antibiotics like; Penicillin $(20 \mathrm{U} / \mathrm{mL})$, streptomycin $(1,00,000 \mu \mathrm{g} / \mathrm{ml})$ or chloramphenicol $(0.2$ $\mathrm{mg} / \mathrm{mL}$ are added to the specimens for preservation. And they can be stored at $4^{\circ} \mathrm{C}$ for no longer than $24 \mathrm{~h}$. However, not all specimens can be frozen. Although refrigerating enables the viability of pathogens and growth of contaminants, blood should be kept at room temperature or perhaps incubated at $35^{\circ} \mathrm{C}$. This goes for CSF as well (room temperature). Organisms like; Hystoplasma capsulatum, Cryptococcus neoformans and Blastomyces dermatitidis cannot grow well in frozen environments. Culture media like brain heart infusion broth is the best medium for transportation of these specimens while ensuring the sample quality [1].

In recent years there are needs to implement more sophisticated methods in detecting these agents of diseases. The reasons are; the multiple species complex nature of fungi and to certain extent the inability to detect at a very low concentration [2-5]. Like the case for bacteria, the non culturable forms of fungal pathogens and the nonsporulating require the need of molecular methods; for example in the 
understanding of biotrophic (those parasites that need its host to survive), endophytic (that lives within a plant for at least part of its life cycle without causing apparent disease) and mycorrhizal type groups of fungi [5].

The reason for an escalating number of molecular techniques in the mycology sectors are but not limited to; The more sensitivity found in the results, the increase output in a short turnaround time, conventional are laborious and took days while molecular method can produce results in hours. The ability to identify and classify agents up to species level and strain types, the ability to detect the molecular mechanisms that are responsible for resistances in the diseases, and molecular typing. If both methods are equivalent in sensitivity, time becomes the choice on the ladder. In addition to the high speed detection, accuracy using molecular methods to detect fungal pathogen, detection based on antigenic and metabolic components is being developed for early and specific diagnosis in the treatment $[1,2]$.

\section{Methods}

\section{Traditional methods}

Although some are outdated and hardly used in the laboratories, however, some classical methods that includes; histopathology, culture and histology (PAS stain) are still in used. For example even with the introduction of molecular methods, PAS stain techniques gives the best result for onychomycoses. Cutaneous fungal infections were traditionally based on microscopic potassiumhydroxide $(\mathrm{KOH})$ preparation and focusing. This technique is sometimes called Blancophor Preparations [6].

Although they serve us in the past, one of the disadvantages of histology and microscopy is the limitations that do not enable them for species identification instead the mere presences of either hyphae or spores. For further identification; both in macro and micro, culture plates are sought for. If not, the weeks-long culturing, and the inability for the agents to grow on the culture media even if the $\mathrm{KOH}$ preparation is effective, molecular methods would have been secondary. However, initial treatment cannot proceed without the preliminary results and the risks involved in week long waiting are prone to even more complications. Although still in the debate, only a highly experienced laboratory technician understands and would be able to classify fungal pathogenic species accurately [6].

\section{Culture methods}

Like bacteria, fungi being a microorganism with distinct metabolism require specific growth and reproduction requirements. Thus, an appropriate growth media is needed to grow the targeted pathogen. The very most common are but not limited to includes; Sabouraud dextrose agar (SDA) and brain-heart infusion agar. Culturing these involves the use of either petri dish or in a tube; both has it advantage over the other. While the disc provides large surface area for accurate colony counting and enabling the collection of pure colony for sub culturing, the tube is user friendly when it comes to transporting to another place. Although the disc after a prolonged incubation might face drought and dried up, it can be moisturized by polyester bad and all edges sealed to avoid air inside.

Multi-purpose growth media that includes; brain heart infusion heart will grow all types of fungal pathogens. In the recent developments, it has been shown that SDA cannot be absolutely relied on for primary recovery medium since some fastidious organisms cannot meet their nutrient requirements [1]. A typical example is Dimorphic Fungi. Potato dextrose agar is now used as a substitute. Again, the excessive nutrient might give over growth of some organism; example is the excessive mycelia growth. This will give counting error while in sporulation. To solve this menace, a combination of both agars gives a better result [1]. Fastidious $B$. dermatiditis is grown in Potato Dextrose Agar. This is normally with $5-10 \%$ sheep blood for the growth of $H$. capsulatum. Antibiotics like; cyclohexamide and chloramphenicol $500 \mathrm{mg} / \mathrm{l}$ and $50 \mathrm{mg} / \mathrm{l}$ respectively are added to reduces contamination with saprophytic fungi [1].

One of the requirements that need special attention when culturing fungal pathogen is the temperature requirements. Most fungi will grow under body temperature range $\left(37^{\circ}\right)$. Molds and Yeasts have different physical appearance on a culture plate and these distinct characteristics made identification very simple and universal. For example; the smooth, creamy, viscous appearance shows yeast while the 7-14 days mold and has a cobweb aerial mycelium and with that, one of the dimorphic agents is suspected [1].

\section{Wet mount or direct microscopic}

Wet mounts dating to ancient techniques, preparation of wet slides using $\mathrm{KOH}$, calcofluor white and India ink played a big role in fungal pathogenic identification. The most common staining procedures until of recent are Giemsa and periodic acid-Schiff (PAS) [1].

\section{Staining}

Staining involves the introduction of dye that enables a clear identification and reading of pathogenic agent from the microscopic slide. A typical example is Fluorescent antibody staining; clinical samples like; blood, CSF, pus, tissue impression smears can be used to detect fungal antigen. This method is still use amongst today's diagnostic tools and even if only few organisms are present, it can be detected. However, like any other methods, sputum analysis is almost always not efficient. Furthermore with the specificity, it is limited to only a spectrum of antisera and for this more sophisticated methods are needed. Another limitation is giving an organism a pseudo pigment and hence it complicates the taxonomy [1].

The views from the microscope are specific to every organism. However, some has similar distinctive morphology. For fungal; if both the yeast and hyphae are present in the field, Candida albicans is suspected. Aspergillus can likely if there are septated hyphae that appear at the angle while if there is no septet, but a broad and ribbon like hyphae structure gives Mucorales [1]. Calcoflour enable clear image since it binds to the wall and make the structure focused through fluorescence microscope. For yeast infections, gram staining is the best option while Giemsa staining for yeast cells of Histoplasma capsulatum. Indian ink creates a dark background and this gives a clear vision for hyaline yeasts cells and capsular material. However, sometimes Indian ink brings confusing in identifying microorganisms as white blood cells.

\section{Serological methods}

Serological methods are categorized under non-culture-based diagnostic tools. Invasive fungal infections (IFIs) can be detected even at an early stage of development; this confers more sensitivity to the non-culture method [1-11]. The principle, just as in bacterial serology is the detection of antibodies or antigen in the serum. The limitations facing includes; cross reactions, the inhibitory effects that can be 
brought by strong immunity; an antibody can be in the tissue but the disease stage was not developed and hence false negative result. Until today, the recognized tools are; immunodiffusion, immuneflourescent antibody, ELISA, agglutination and western blotting/ immunoblotting.

\section{Molecular techniques}

Just as the technological advancement proceeds in other sectors; the introduction of new diagnostic tool in mycology also follows the same trend. With the coming of molecular techniques, infectious diseases diagnostics has experienced a level of identifying DNA or the fingerprint of pathogenic agents. One of these methods that are now common in the routine laboratories is Polymerase Chain Reaction (PCR). This brought a boost to the identification of human diseases. Of course their reliability, sensitivity and specificity put them before the traditional methods [1-12].

\section{Fungal DNA extraction}

Prior to any molecular identification based on nucleic acid techniques, DNA or RNA extraction is very crucial and inevitable. The method employed and the technical know-how to extract the pathogenic DNA determines the purity of sample to be tested. In these days of commerce, many tools are used with the aim of extraction the DNA from fungal sources. Apart from the manual steps that has a long history of extracting fungal DNA; commercial kits are also in numbers. However, the questions are; if these kits really specific and reliable? Until today, there are no extraction kits for soil fungal pathogens and this has created a giant obstacle. Paint shakers are used for soil samples. Again at some instances, even when the kits are specific, during the process of isolation and extraction, cells can be disrupted and this can potentially affects the nucleic acid quantity and quality. This can be achieved through the co extraction of humic acids that is an inhibitor of PCR or by the direct shearing of the nucleic acid. Also the choice of the buffer used might hinder the homogenization of cells. According to many fungal pathologists, $\mathrm{CTAB}$ or guanidinium thiocyanate based buffer is most appropriate [2-10]. In response to the need of attaining pure form of DNA, there are number of procedures that can assure the quality and quantity of DNA. Among these are silica membrane spin fiber, slilcamagnetic and pelleting. Furthermore the choices of these methods depend entirely on the type of amplification and sequencing system that will be taken and perhaps the cost involved is crucial. A typical example is; while real time PCR for routine purposes does not require a highly pure nucleic acid; an absolute purity is required for the sequencing with the use of Highthroughput sequencing [5].

\section{Polymerase chain reaction (PCR)}

Since its coming to the molecular world, PCR has its roots in amplifying the nucleic acid that enable the technicians to identify specific pathogen agents. Its principle is in three stage process; denaturation, annealing and extension on different temperature scales. All of specific primers, probes or dyes, template, buffer solution and salts are added alongside with enzyme to produce a master mix. Initially, the use of gel electrophoresis was used. However due to nonquantification of the amplicon, real time PCR is preferred. One of the most cares taken procedures in PCR diagnostics is the isolation of DNA. The purer the nucleic acid, the more accurate the results will be. In mycology, molds normally require cumbersome and complex process before acquiring their DNA. This is due to the rigidity of their cell wall [2-5]. Phenol-chloroform chemical is used to digest the cell wall and care must be taken. In addition, automated extraction kits yield even purer DNA but its activity in digesting cell wall is not assured.

One of the limitations of PCR is to identify stages of disease; for example the colonization and infective stage of Aspergillus spp. isolation. Technical errors and the selection of specific primers for the IF pathogen detection are factors, this due to the closeness of the segments of the gene to be amplified. [9] Even with the most molecular similar structured organisms like F. acuminatum spp., F. graminearum, F. pseudograminearum and F. avenaceum spp. PCR is capable of identifying with accuracy One of its success story on sensitivity is on Zymoseptoria tritici and Blumeria graminis $f$ spp. Tritici [11]. These plant pathogens can be detected even in a latent period [5].

Internal transcribed spacers (ITS) are one of the most successful PCR based techniques. These methods imply the use of both ITS1 and ITS2 on a specific segment of a fungal gene for identification; around the 5.8S-coding sequences on the fungal genome. Since the ITS region of the gene is very stable, conservative within the species and occurs in multiples, the exact section can be easily found between $18 \mathrm{~S}$ of the small subunit of the ribosome (SSU) and the large subunit $28 \mathrm{~S}$ (LSU). Just like in bacterial primer design, universal primer set are as well designed that are specific in binding to the targeted or conserved region of gene that should be amplified. These identifications are either through DNA sequence or the use of restriction analysis of amplicon. As of today, a universal database has been developed by ITS sequence that gives a highly identification and shows relativeness up to $3 \%$ of gene similarities. However, the genetic identification of this method is said to be insufficient [5].

Other methods include; intergenic spacer (IGS), protein coding genes, ribosomal DNA (rDNA), random amplified polymorphic DNA (RAPD) and mitochondrial DNA (mtDNA).

$18 \mathrm{~S}, 5.8 \mathrm{~S}$ and $28 \mathrm{~S}$ of rDNA have both variable and conserved regions that are highly species-specific primer design. They are most efficient when it comes to distant the identification of organism that has distant taxonomic [5]. The D1 and D2 domains found in the 5'end in $28 \mathrm{~S}$ of rDNA gene have the ability to identify monophyletic groups based on the secondary metabolite production [5].

Conditions in which ITS regions show lower variability, the IGS or non-transcribed is useful, this is due its location (between 28S (LSU) and $5 \mathrm{~S}$ in IGS1 while in IGS2, the locations are immediate right of $5 \mathrm{~S}$ towards the 3 ' end). In the sequencing using trichothecene, genes like Tri3, Tri5 and Tri6 are preferred. Although it's A+T content is $90 \%$ and that makes it difficult to amplify, mtDNA has its advantages when it comes to its high copy number, the presences of conserved and variable regions and its small size [5].

In this world of molecular technology, several versions of PCR come in with unique effectiveness in pathogen detection. Among these are; quantitative PCR or real time PCR. Its sensitivity and the ability to show amplicon in real time gives its advantage over conventional PCR tools. However, with the fungal PCR, the real time PCR might only come second to conventional PCR in case where the DNA is insufficient or the rigid cell wall of species like; $M$. graminicola that would hardly allow their cell wall to disrupt in order to give its nuclear material.

Fluorescent amplification based specific hybridization (FLASHPCR) is a PCR method that uses probe hybridization with fluorescent to identify pathogens. It is another version that is semi real time 
(results are only read after termination stage) and above conventional PCR (it does not require gel electrophoresis). P. nodorum, toxigenic Fusarium spp. and $Z$. tritici were identified accurately through FLASPCR [11]. Another method that shows greater positive results even if the DNA is not highly purified is Loop-mediated isothermal amplification. However, when dealing with environmental samples, foreign nucleic acids are paramount and this leads to false positive results [5] (Table 1).

\begin{tabular}{|l|l|}
\hline 18S rDNA & 508,808 \\
\hline ITS including 5.8S & 181,258 \\
\hline $\boldsymbol{\beta}$-Tubulin & 59208 \\
\hline IGS1 and IGS2 & 1,524 \\
\hline Tri5 454 & 454 \\
\hline Calmodulin & 24298 \\
\hline 28S rDNA & 423,206 \\
\hline EF-1- $\alpha$ & 21546 \\
\hline
\end{tabular}

Table 1: Selected fungal sequences published in the NCBI Gen Bank.

Multiplex PCR brings in simultaneous detection of pathogens or targeted genome segments goes as far to fungi. This procedures is as simple as the singleplex, however the principles lies entirely on the selection of specific primer. The understanding of the primer and probes is highly necessary and also the differences in the melting point range. Melting curve analysis is use to determine the level of contamination of the amplicon. Multiplex has already achieved its success story in the detection and amplifying fungal agents in whole blood cells, serum or BAL fluid samples from Invasive Fungal Infections (IFIs) patients. SetiFast assay is a commercially developed kit to detect sepsis occurrence pathogens [1,2]. Another version of PCR in the multiplex family is the Multiplex-tandem PCR (MT-PCR). This deals with highly multiplexed gene expression profiling and quick identification of pathogens. The amplicon of the first step normal multiplex PCR is diluted to serve as the template to be amplified in the second step. SYBR green is the dye and hence melt-curve analysis to rule out the contaminants. Due to the demanding needs to address the situations of bloodstream infections, MT-PCR assays are develop and these targets are but not limited to; 11 species of Candida, Saccharomyces cerevisiae, F. solani, pan Fusarium, Scedosporium prolificans and Cryptococcus neoformans, complex. ITS squeence variations were used to designed primers, also used are; elongation factor 1-a (EF1-a) and b-tubulin genes [5].

\section{S rRNA}

Similar to the systematic classification by Bergey's manual of Systematic Bacteriology for Pseudomonas, 28S rRNA gene is still a criterion for the diversity order in fungal. This method classified bacterial genus and in almost in all the standard mycology laboratories, a sequence database that is web-based has been generated. This has the ability to benchmark up to millions of fungal sub species. In the history, the macromolecules containing cytochrome $c$ and ribulose bisphosphate carboxylase (distant taxa) were used in drafting the phylogeny of fungal. However due to the differences in metabolism and chemical components, these has encountered lots of setback for non-producing microorganisms. Among the three RNA types, (5S rRNA, $23 \mathrm{~S}$ rRNA and $28 \mathrm{~S}$ rRNA), the $28 \mathrm{~S}$ rRNA stands unique with the amplification by PCR and gene sequencing analysis. It is still the reference gene for fungal taxonomy. Its universal in nature enable even a single pair of primer to identify targets on the gene segments and this boost its usage and hence an advantage over culture based fungal identification. Similar to bacterial molecular identification, DNA is needed to be extracted, quantified in the Nanodrop, design primers, amplify and analyzed the amplicon with references to the database, Ribosomal Database Project (RDP). This probe match enables a quick search in the database to check primer and probe coverage up to 60 bases in length [5].

Another breakthrough in the annals of mycology is The Blast Local Alignment Search Tool (BLAST). This encompasses wide range of applications; both nucleotide and protein are amplified. This method is basically used to solve queries that arise from 28S rRNA through the NCBI bioinformatics program [3]. Terminal-restriction fragment length polymorphism is also another tool that is used to study fungal diversity and this is based on 28S rRNA gene. Even with multiple primers, the use of fluorescence, fragments are accurately separated in the electrophoresis [3]. Furthermore, the use of temperature gradient gel electrophoresis, sometimes referred to as denaturing gradient gel electrophoresis relies on denaturing properties of double-stranded $28 \mathrm{~S}$ rRNA. The variation of these denaturing temperatures corresponds to 28S rRNA gene sequencing [5].

\section{Fluorescence in situ hybridization (FISH)}

In a bid to enhance the producing of quality result, PCR related methods are used as a supplement. One of these is FISH. This technique uses fluorescent probe that enable a clear view of the genome segment that is focused through microscope. Its ability to differentiate between real players (active growing organism) from other dormant organisms in environmental samples gives its novelty in the lights of molecular tools. In the presences of RNA, the need for culture becomes secondary since FISH has the ability to clearly visualize the exact location of nucleic acids in organelles. Due to its abundance in the database and multiple copies of cells, FISH targets rRNA and mitochondrial genes. The fungal and substrate inherent auto fluorescence (false positive results), non-specific binding of probes, low ribosome contents and insufficient permeability of cell wall account for the limitations of FISH and for cell wall impermeability, a common method to prevent it is the use of cell wall lysing enzymes such as chitnases and glucanases. In addition, peptidenucleic acid (PNA) probe is also used. This mimics the negatively charged sugarbackbone of DNA while replacing it with non-charged polyamide backbone [12]. Catalysed Reporter Deposition FISH (CARD-FISH) is a version of FISH that enchance the singals of probe. Herein the probes are labeled with horseradish-oxide in the place of flourochrome and after hybridization, fluorescence labeled tyramide are added to the cells [5].

\section{MALDI-TOF MS}

This method is based on mass spectrometry in detection and identification of protein finger prints. Depending on the spectra, the organism can be easily identified after matching with the Database that goes beyond genus, species to strain typing. Matrix-assisted laser desorption ionization-time of flight mass spectrometry (MALDI-TOF MS) is used in conjunction with the broad-range PCR that targets rDNA fragment. This enables the identification of pathogens from the specimens of patients and also methylprednisolone vials [2-8]. MALDI 
has shown increasing positive results for the rapid diagnosing Candida spp. and Aspergillus spp. and this give its advantage over the corresponding traditional methods. MALDI-TOF MS is also an accurate and rapid technique for identification of spoilage.

Recently, there is even a new version of MALTOF that does not required protein extraction step instead using single deposit step and this prevents the lysis of formic acid. The Vitek MS system is an example of the newer version and it makes the method user friendly [2-10].

\section{Nucleic acid sequence-based amplification (NASBA)}

A very similar method to PCR, the only differences is their approaches. Instead of DNA, it amplifies RNA by using RNA polymerase instead of DNA polymerase and also it is isothermal (this helps to decreases contaminants since RNA is less stable and can stabilize at a temperature lower than that of DNA). Since it detects RNA, not DNA, only active pathogenic agents are detected. This is a very big advantage over PCR [2].

\section{Nuclear magnetic resonance (NMR) spectroscopy}

This method is based on physical phenomenon in identifying fungal pathogens. For over 70 years, it has registered its proficiency in various disciplines like chemistry, biochemistry etc. However, it is just of recent that microbiology incorporates this method and mycology to be more specific. It is a potential method to identify Candida spp. especially those isolated from blood culture specimens $[11,12]$.

\section{Micro array}

In one of the recent studies, it is proven that with the microarray, the turn-around time is much quicker for the identification of Saccharomyces cerevisiae. This means a microarray is a potential tool for rapid detection and identification of yeast infections like; Candida species [10].

\section{Restriction fragment length polymorphism (RFLP)}

RFLP is one of the methods that involve the use of enzyme digestion of DNA and this is read through gel electrophoresis using agarose or polyacrylamide gels based on the size of the DNA fragments. Using this technique, a foreign DNA that is oftentimes pathogenic is easily detected. The restriction enzyme has in it a polymorphism that cleavages specific site in the gene segment that is relative to distinguish fungal species [11].

\section{Random amplified polymorphic DNA (RAPD)}

Analyses entirely rely on PCR amplification of the pathogen genome with short arbitrary sequences. These are used as primers. This is a very useful tool used for genetic diversity among fungal population. A typical example is to analyze the genetic diversity among different species of Fusarium spp. and different pathotypes of Elsinoë spp. However, one of its limitations is its inability to differentiate homozygotes and heterozygotes individuals and due to this, the differentiation of basidiomycetes and oomycetes that are heterokaryons, diploids or polyploids becomes an issue [11].

\section{Amplified fragment length polymorphism (AFLP)}

With this method, a total genomic DNA is digested by restriction enzyme. Here, the digestion is preceded by the ligation of restriction half-site specific adapters that is peculiar to all fragments. This is followed by amplification of PCR primers while the 3 prime end corresponds the sequence. It is to distinguish Cladosporium fulvurn from Pyrenopeziuz brassicae species. Also, Aspergillus carbonarius from A. ochraceus and Colletotrichum gossypii from C. gossypii var. cephalosporioides. Like other tools, one of its setbacks is its requirement of high DNA weight and involves a high technical knowhow [11].

In addition to aforementioned tools, Microsatellites, also known as simple sequence repeats (SSRs) or short tandem repeats (STRs), Allelespecific PCR and quantitative allele-specific real-time PCR and PCR, PCR-RFLP and primer-introduced restriction analysis PCR (PIRAPCR) detection of DMI-resistant isolates many other tools that are not mentioned are equally promising tools in identifying Fungal pathogens [11-13].

\section{Conclusion}

Like the common the saying, there is a solution to every problem, so due to the occurrence of fungi infections. Coming to the new generation, the misconception of fungal disease and these outbreaks are now defined and these enable the pathologist to understand the geographical limitations of specific species and strain type. Although there will be a continuous appearing and re-emerging of new species in the human world, the accurate diagnosis of these outbreaks will enable the mycologists to prepare even better. With the coming of gene sequencing technologies, the misconceptions that were related to the classification of fungi based on their phenotypic appearances and their reproductive preferences is fading away.

In the recent times, most of these methods can only be found in highly advanced laboratories. Collection of appropriate clinical specimens determines the success of fungal pathogen diagnosis. In the level of molecular test, selecting the most effective methods also ensures accuracy in diagnosis. The general practical approaches in mycology are; methods of collecting specimens, either direct or staining, culture on selective media or some non-culture based [1].

However, soon they will secure there functions in the daily routine laboratories. The most critical issues regarding the new methods are validation. Various analytical tools are tested before incorporated into the diagnostic laboratories. All these parameters should favour the sensitivity, specificity and accuracy of a particular method $[1,2]$.

\section{Conflict of Interest}

There is no conflict of interest.

\section{References}

1. Mulla (2015) Lab diagnosis: Fungal infections Universal Research. J Dent $5: 2$.

2. Arvanitis Anagnostou T, Fuchs BB, Caliendo AM, Mylonakis E (2014) Molecular and non molecular diagnostic methods for invasive fungal infections. Clin Microbiol Rev 27: 490-526.

3. Srivastava M (2014) To develop strain specific molecular marker for easy and proper identification of fungal species based on molecular characters: A review J Mol Biomark Diagn 5: 172. 
Citation: Bajinka O, Terzi Y, Ucar F (2017) The Development of Diagnostics Tools and Techniques in the Isolation and Detection of Fungal Pathogens. J Infect Dis Med 2: 119. doi:10.4172/2576-1420.1000119

Page 6 of 6

4. Guarro J, Gené J, Stchigel AM (1999) Developments in fungal taxonomy. Clin Microbiol Rev 12: 454-500.

5. Tsui CKM, Woodhall J, Chen W, Lévesque CA, Lau A, et al. (2011) Molecular techniques for pathogen identification and fungus detection in the environment. IMA Fungus 2: 177-189.

6. Ali MA, Xi J, Coudray J (2015) Comparisons of molecular methods in the diagnosis of pathogenic fungi. International Journal Of Scientific \& Technology Research 4: 234-236.

7. Kuzdraliński A, Kot A, Szczerba H, Nowak M, Muszyńska M (2017) A review of conventional PCR assays for the detection of selected phytopathogens of wheat. J Mol Microbiol Biotechnol 27: 175-189.

8. Lima N, Santos C (2017) MALDI-TOF MS for identification of food spoilage filamentous fungi. Curr Opin Food Sci 13: 26-30.

9. Capote N, Pastrana AM, Aguado A, Sánchez-Torres P (2012) Molecular tools for detection of plant pathogenic fungi and fungicide resistance. Plant Pathology. InTechOpen, London. pp: 151-202.
10. Farina C, Russello G, Andreoni S, Bonetti C, Conte M, et al. (2012) Microarray technology for yeast identification directly from positive blood cultures. A multicenter Italian experience. Med Mycol 50: 549-555.

11. Wiegand C, Bauer A, Brasch J, Nenoff P, Schaller M, et al. (2016) Are the classic diagnostic methods in mycology still state of the art? J Dtsch Dermatol Ges 14: 490-494.

12. Kondo T, Terada K (2017) 72-year-old man presented with a 2-week history of odynophagia (pain on swallowing). N Engl J Med 376: 16.

13. Angeletti S (2017) Matrix assisted laser desorption time of flight mass spectrometry (MALDI-TOF MS) in clinical microbiology. J Microbiol Methods 138: 20-29. 